\title{
Linfoma no Hodgkin primario de sistema nervioso central en un paciente pediátrico sin inmunodeficiencia relacionada
}

\section{RESUMEN}

El linfoma no Hodgkin primario de sistema nervioso central es una afección poco frecuente en la población pediátrica. No existen (o son pocas) las recomendaciones o estudios clínicos que sean útiles para definir la mejor opción terapéutica. El objetivo de este trabajo es describir el caso de una niña con linfoma primario de sistema nervioso central, sin ninguna inmunodeficiencia asociada, y realizar de una revisión cualitativa de la literatura especializada sobre esta enfermedad en niños.

Se ha postulado la posibilidad de cambios citogenéticos que pudieran explicar la heterogeneidad en el comportamiento de esta enfermedad pues se ha visto que es distinto al de otros linfomas en presentaciones habituales. La experiencia de tratamiento en niños con linfoma se basa en resultados de tratamientos obtenidos en pacientes adultos, con regímenes basados principalmente en antimetabolitos como metotrexato y citarabina. Aún es controvertida la utilidad de la radioterapia craneal, la cual sólo se recomienda en casos específicos.

Palabras clave: linfoma, sistema nervioso central, pediatría, quimioterapia.

\section{Primary central nervous system non Hodgkin lymphoma in a pediatric patient without related immunodeficiency}

\begin{abstract}
The central nervous system primary lymphoma is a very infrequent disease in the pediatric population. Currently there are no or few recommendations and/or clinical useful trials to define the best therapeutic option in this population. The objective is to report the case of a child with primary lymphoma of the central nervous system without any associated immunodeficiency, and to do a qualitative review of the literature regarding this disease in children. The possibility of cytogenetic changes has been postulated explaining the heterogeneity in the behavior of this disease, since it has been seen that it is different from other lymphomas in usual presentations. The experience of treatment in pediatric patients with lymphoma is based on results obtained in adult population, mainly with the use of anti-metabolites such as methotrexate and citarabine. The use of cranial radiation therapy is still controversial and is recommended only in special cases.
\end{abstract}

Key words: Lymphoma, Central nervous system, chemotherapy
Rocío Cárdenas-Cardos ${ }^{1}$

Marco Rodrigo Aguilar-Ortiz ${ }^{2}$

Liliana Velasco-Hidalgo ${ }^{2}$

Pedro Mario Pasquel García-Vázquez ${ }^{3}$

Roberto Rivera-Luna ${ }^{4}$

Alfonso Marhx-Bracho ${ }^{5}$

${ }^{1}$ Médico oncólogo pediatra, jefa del Servicio de Oncología Médica Pediátrica.

${ }^{2}$ Médico oncólogo pediatra.

${ }^{3}$ Médico patólogo.

${ }^{4}$ Médico hematooncólogo pediatra, subdirector de Hemato/oncología.

${ }^{5}$ Jefe del Servicio de Neurocirugía Pediátrica.

Instituto Nacional de Pediatría, Ciudad de México.

Recibido: 28 de agosto del 2014

Aceptado: 25 de febrero del 2015

Correspondencia: Marco Rodrigo Aguilar-Ortiz Instituto Nacional de Pediatría Insurgentes Sur 3700-C CP 04530 México, D.F.

Tel. 10840900 ext. 1343

marcorodrigo@yahoo.com

Este artículo debe citarse como

Cárdenas-Cardos R, Aguilar-Ortiz MR, VelascoHidalgo L, Pasquel García-Vázquez PM, Rivera-Luna R, Marhx-Bracho A. Linfoma no Hodgkin primario de sistema nervioso central en un paciente pediátrico sin inmunodeficiencia relacionada. Acta Pediatr Mex 2015;36:389-396. 


\section{INTRODUCCIÓN}

De acuerdo con la Organización Mundial de la Salud los linfomas primarios del sistema nervioso central son el grupo de neoplasias malignas de origen linfoide extranodal primarias del sistema nervioso central, en ausencia de enfermedad fuera del sistema nervioso central al momento del diagnóstico, ${ }^{1}$ que se pueden presentar en encéfalo, leptomeninges, médula espinal u ojos. ${ }^{2,3}$

Esta anomalía es sumamente rara en la infancia y se desconoce su incidencia pero distintas series señalan que representa de 1 a $1.5 \%$ de los linfomas no Hodgkin en menores de 19 años y se estiman de 15 a 20 casos al año en Norteamérica. ${ }^{4}$ Se ha descrito que representan aproximadamente de 0.5 a $2 \%$ de los tumores primarios del sistema nervioso central en niños ${ }^{3}$ pero son pocos los estudios en esta población que proporcionen información del comportamiento de esta enfermedad y la mayor parte se deriva de series pequeñas o es extrapolada de estudios en adultos. ${ }^{5,6} \mathrm{La}$ edad de presentación promedio descrita al diagnóstico es en pacientes adolescentes con una media de 14 años, con mayor frecuencia en el sexo masculino. ${ }^{1}$ El principal factor de riesgo identificado en la biología de estos linfomas es la asociación con inmunodeficiencias congénitas o adquiridas, así como con infección por virus de Epstein-Barr; la mayoría de los casos reportados eran pacientes inmunocompetentes. . $^{1,2,4,5,7}$

Estos tumores se clasifican como linfomas no Hodgkin de acuerdo con la clasificación de la Revised European-American Lymphoma (REAL) y con la clasificación de la Organización Mundial de la Salud, la mayoría es de linaje B. En niños la histología más frecuentemente descrita de linfomas primarios del sistema nervioso central es el linfoma difuso de células grandes B que representa hasta $69 \%$ de los casos; ${ }^{1,2,4,5,8}$ en segundo lugar el linfoma anaplásico de células grandes $T$ con $17 \%{ }^{4}$ y, por último, los linfomas linfoblástico y similar a Burkitt con $7 \%$ cada uno. ${ }^{4}$

Históricamente el linfoma no Hodgkin se ha asignado a grupos de alto riesgo para su tratamiento debido a su localización primaria. ${ }^{9-15}$ Las estrategias terapéuticas se han basado en radioterapia (craneoespinal), quimioterapia e incluso trasplante de células madre hematopoyéticas. La cirugía sólo se limita a la obtención del material suficiente y adecuado para el diagnóstico. ${ }^{4,5,8-10,15-19}$

Actualmente no existe un esquema terapéutico ideal para población pediátrica. Los informes de casos en adultos han mostrado supervivencia a largo plazo sólo con quimioterapia. La serie pediátrica más grande sugiere que la mayoría podría lograr remisión a largo plazo sólo con quimioterapia, sin radioterapia craneoespinal; No obstante, dichos estudios son limitados debido a que las series de pacientes eran muy pequeñas $4,5,16,17,19$ (Cuadro 1). El pronóstico es pobre, se ha informado supervivencia libre de eventos a 5 años desde 25 hasta $40 \%$; entre la población pediátrica dicha supervivencia es de hasta $70 \%$ pero con una tasa alta de recaídas. ${ }^{1,2,4,5,15-17}$

\section{OBJETIVO}

Describir el caso y evolución clínica de una niña con linfoma primario del sistema nervioso central, debido a que se trata de una afección infrecuente en pacientes pediátricos, así como relatar el comportamiento agresivo de este caso que tuvo un especial interés para la adecuación del tratamiento. Se realizó un análisis cualitativo de la información reportada en la literatura especializada.

\section{CASO}

Niña de diez años, diez meses de edad, cuyo padecimiento tenía un mes de evolución e inició 
Cárdenas-Cardos $\mathbf{R}$ et al. Linfoma no Hodgkin primario de sistema nervioso central

Cuadro 1. Estudios pediátricos de linfomas primarios del sistema nervioso central

\begin{tabular}{|c|c|c|c|c|c|c|}
\hline Autor & Año & Pacientes & Edad (rango) & Histología & Tratamiento & Supervivencia genera \\
\hline Abla O & 2006 & 12 & $7.5 a(4-12 a)$ & $\begin{array}{c}7(58.3 \%) \text { difuso de } \\
\text { células grandes } \\
4(33.3 \%) \text { anaplásico } \\
1(8.3 \%) \text { Burkitt }\end{array}$ & $\begin{array}{c}\text { Sólo QT (altas dosis de } \\
\text { MTX y CT) } 10(83.3 \%) \\
2(16.6 \%) \text { RT } \\
12(100 \%) \text { TIT }\end{array}$ & $\begin{array}{c}5 \text { años } \\
70 \pm 14.5 \%\end{array}$ \\
\hline Abla $\mathrm{O}$ & 2011 & 29 & $14 a(2-21 a)$ & $\begin{array}{c}20(69 \%) \text { difuso de } \\
\text { células grandes } \\
5(17 \%) \text { anaplásico T } \\
2(7 \%) \text { linfoblástico } \\
2(7 \%) \text { Burkitt }\end{array}$ & $\begin{array}{c}\text { Sólo QT } 18(62 \%) \\
\text { QT + rituximab } 2(7 \%) \\
\text { QT + RT } 9(31 \%)\end{array}$ & $\begin{array}{c}3 \text { años } \\
82 \% \text { IC } 61-92 \%\end{array}$ \\
\hline Hyung JY & 2012 & 6 & $\begin{array}{c}10 a 1 m \\
(23 m-11 a \\
9 m)\end{array}$ & $\begin{array}{l}3 \text { difuso de células } \\
\text { grandes } \\
2 \text { Burkitt } \\
1 \text { B indiferenciado }\end{array}$ & Sólo QT & $\begin{array}{c}\text { Estimada a } 5 \text { años } \\
83.3 \%\end{array}$ \\
\hline
\end{tabular}

QT: quimioterapia; MTX: metotrexato; CT: citarabina; RT: radioterapia; TIT: terapia intratecal; IC: intérvalo de confianza.

con vómito y ataque al estado general, se le trató como enfermedad acidopéptica sin mejoría. Tiempo después, la paciente presentó alteración de la marcha, por lo que se realizó tomografía axial computada de cráneo que mostró una zona hiperdensa en el cuarto ventrículo, que medía $2.5 \times 3.5 \mathrm{~cm}$ y desplazaba rostralmente al tallo cerebral (Figura 1) La resonancia magnética mostró una lesión del cuarto ventrículo hipointensa homogénea, extraaxial, que reforzaba de forma difusa al contraste (gadolinio); sin infiltración aparente del cuarto ventrículo ni del pedúnculo cerebeloso por lo que se sospechó diagnóstico de meduloblastoma. (Figura 2) Lo anterior llevó a decidir una resección quirúrgica de la lesión en un 90\% aproximadamente. Un informe transquirúrgico de patología informó de un tumor de células pequeñas, redondas y azules (Figura 3a). El informe histopatológico final fue de neoplasia maligna de estirpe linfoide con marcadores inmunohistoquímicos positivos para CD-45 (Figura 3b) y CD-20 (Figura 3c), base para el diagnóstico de linfoma difuso de células grandes B.

Como parte del estudio diagnóstico se efectuaron varios análisis para descartar inmunodeficiencia primaria o secundaria, asociada con el diagnósti-

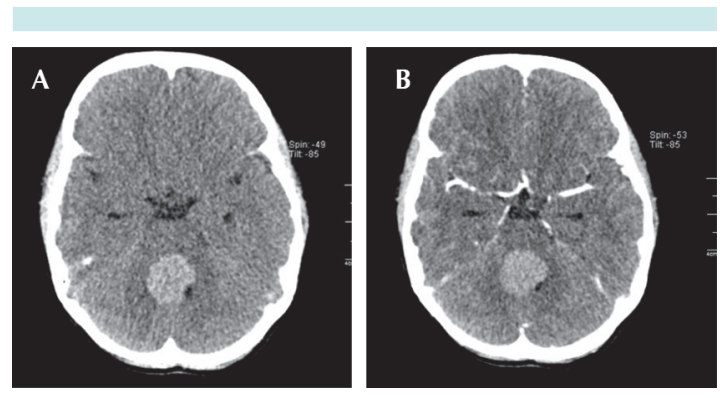

Figura 1. Tomografía computada; reconstrucciones axiales A) Fase simple: lesión en el interior del cuarto ventrículo, de contornos regulares, homogénea, hiperdensa. B) Fase contrastada: realce de forma leve con administración de medio de contraste (60 UH).

co emitido. Determinación de inmunoglobulinas séricas lgG: $915 \mathrm{mg} / \mathrm{dL}$; IgM: $157 \mathrm{mg} / \mathrm{dL}$; IgA: $136 \mathrm{mg} / \mathrm{dL}$ y determinación por ELISA del virus de la inmunodeficiencia humana (VIH) negativo. Para determinar la extensión de la enfermedad de base se realizó una biopsia de médula ósea que mostró hipoplasia leve sin fibrosis reticulínica, negativa para infiltración neoplásica. Un gammagrama óseo posquirúrgico con tecnecio-99 mostró discreta hipercaptación difusa en la región occipital, compatible con remodelación ósea; el gammagrama con galio-67 fue normal. 

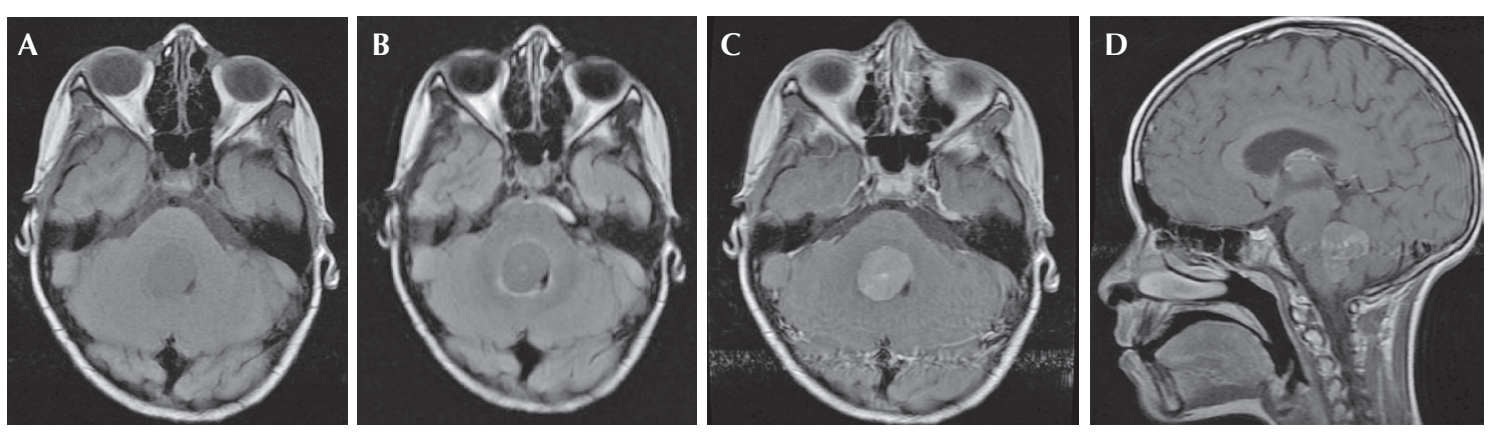

Figura 2. Resonancia magnética. A) Estudio simple en fase T1, corte axial: lesión intraventricular isointensa que condiciona obliteración parcial de la luz del cuarto ventrículo, así como compresión de puente y bulbo raquídeo. B) Estudio simple en fase T2 FLAIR, corte axial. C) Estudio contrastado en fase T1, corte axial: realce de forma homogénea posterior a la administración del medio de contraste. D) Fase T1, corte sagital con medio de contraste.
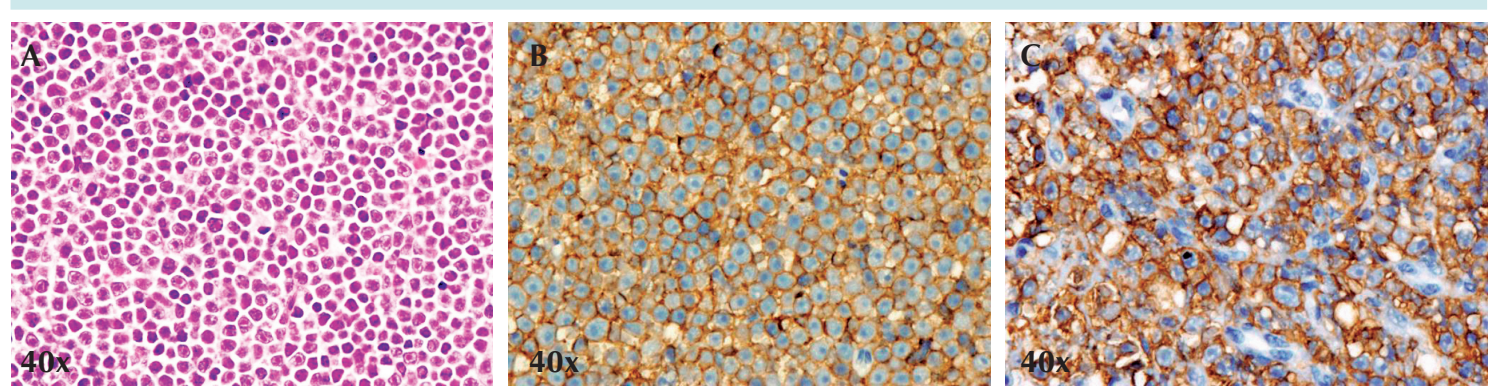

Figura 3. Cortes histológicos. A) Tinción de hematoxilina-eosina: neoplasia maligna de estirpe linfoide que crece en láminas sólidas, células de tamaño mediano a grande, escaso citoplasma, núcleos ovales, cromatina abierta, nucléolo prominente; estas células alternan con linfocitos maduros residuales. B) CD45 (antígeno común leucocitario) positivo intensa y difusamente decorando la membrana celular. C) CD20 positivo intensamente en la membrana de los linfocitos B.

La tomografía por emisión de positrones con fluorodesoxiglucosa mostró una lesión infratentorial hiperdensa de $15 \times 11 \mathrm{~mm}$ localizada en el cuarto ventrículo, con aumento de la actividad glucolítica y valor máximo estandarizado de captación $\left(\mathrm{SUV}_{\text {máx }}\right)$ de hasta 12.7. El resto del rastreo fue normal (Figura 4).

Se inició tratamiento de quimioterapia con fase de citorreducción similar al linfoma no Hodgkin de células $\mathrm{B}$. 9,10,20 Al final de esta fase la paciente tuvo datos de hipertensión intracraneana que se clasificó como enfermedad estable. Se continuó el tratamiento de intensificación ${ }^{20,21}$ con adriamicina y dosis altas de metotrexato, a pesar de lo cual la paciente mostró progresión de la enfermedad por lo que se inició la fase de consolidación (similar al CYVE 1$)^{10}$ que se modificó de acuerdo con las condiciones clínicas de la paciente. El tratamiento quimioterapéutico se resume en el Cuadro 2.

La evolución clínica durante el tratamiento fue tórpida, presentando datos clínicos y radiográ- 

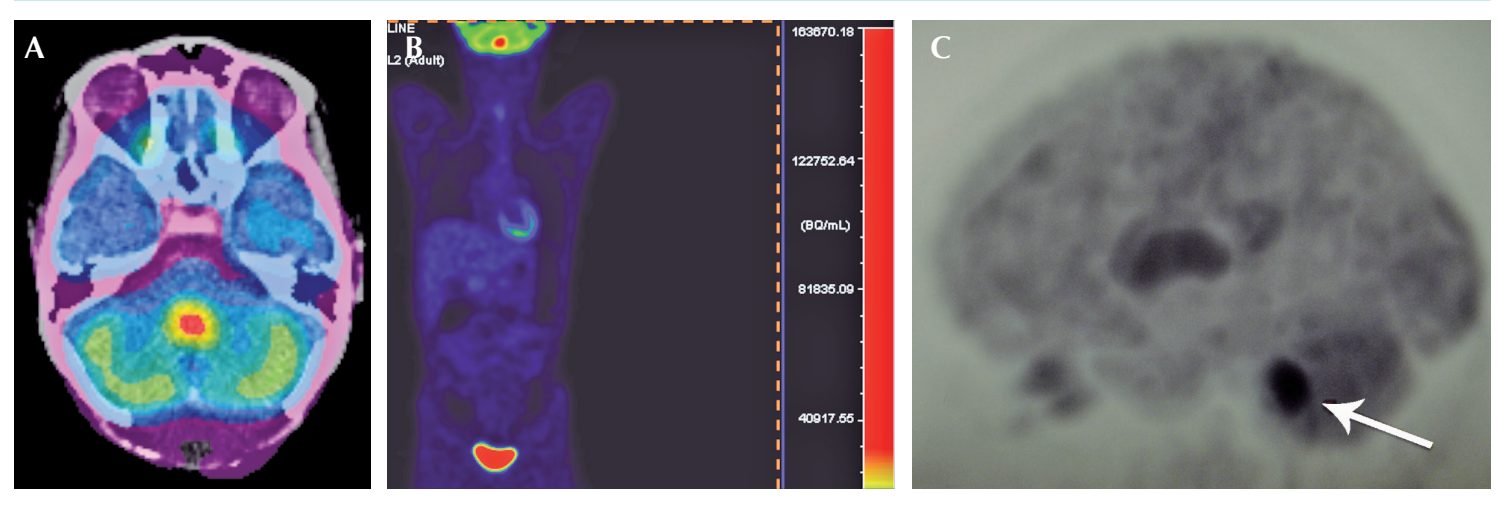

Figura 4. Tomografía por emisión de positrones. A) Reconstrucción axial: aumento de metabolismo $\mathrm{SUV}_{\text {máx }}$ 12.7. B) Proyección cuerpo entero con aumento del metabolismo en fosa posterior, eliminación normal del marcador radioactivo en la vejiga urinaria. C) Proyección sagital del cráneo: aumento del metabolismo en fosa posterior (flecha).

ficos de disfunción del sistema de derivación ventriculoperitoneal y progresión tumoral (Figura 5), por lo que fueron necesarias distintas intervenciones como el recambio del sistema de derivación e incluso se decidió iniciar radioterapia craneoespinal (25 Gy/15 Gy). A pesar de estas intervenciones continuó con datos de progresión tumoral que requirieron cambiar a un esquema de quimioterapia teóricamente con mayor penetración al sistema nervioso central (similar al FAB/LMB-96) ${ }^{20}$ agregando una nueva fase de intensificación basada en dosis altas de metotrexato y citarabina.

La paciente continuó con mala evolución clínica y radiológica; requirió hasta cuatro recambios valvulares por disfunción y datos de hipertensión intracraneal; además había datos de toxicidad hematológica (neutropenia grado IV) e infecciosa relacionada con el tratamiento. La niña falleció con datos de progresión tumoral y sepsis a los seis meses del diagnóstico.

\section{ANÁLISIS}

Se realizó una búsqueda exhaustiva de la literatura en las siguientes bases: Medline 1950-2014
(OVID), Embase 1980-2014 (OVID), LILACS (1998 a 2014), ARTEMISA (1999 a 2014) y SCIELO (1999 a 2013).

El linfoma no Hodgkin primario del sistema nervioso central es una afección poco frecuente en la edad pediátrica, son pocas las recomendaciones y no existen estudios clínicos que puedan apoyar para definir la mejor opción terapéutica. La mayoría de las series revisadas coinciden en la utilidad de las dosis altas de metotrexato $\left(>1 \mathrm{~g} / \mathrm{m}^{2}\right)^{2,4,5,8-12,15-18,21}$ como el agente más eficaz para tratar estos casos. En artículos de población adulta se han informado tasas de remisión de 30 a 65\% (supervivencia libre de eventos de 13-17 meses). ${ }^{2,8,11,16}$ Otros trabajos mencionan mayor riesgo de neurotoxicidad asociada, especialmente en pacientes ancianos, cuando se utiliza en combinación con radioterapia craneal. ${ }^{2,8,11}$ Respecto a la utilidad del metotrexato intratecal se señala que deben utilizarse en combinación con dosis altas de metotrexato para lograr mayor penetración al sistema nervioso central; sin embargo, no ha demostrado aumentar la supervivencia. Cuando la dosis de metotrexato IV es $>3 \mathrm{~g} / \mathrm{m}^{2}$ algunos estudios sugieren evitar la administración in- 
Cuadro 2. Esquema del tratamiento administrado

\begin{tabular}{|c|c|c|}
\hline Tratamiento & Dosis & Tiempo \\
\hline \multicolumn{3}{|c|}{ Citorreducción (COP) } \\
\hline CFM & $300 \mathrm{mg} / \mathrm{m}^{2} \mathrm{IV}$ & Día 1 \\
\hline PDN & $60 \mathrm{mg} / \mathrm{m}^{2} \mathrm{VO}$ & Días 1-7 \\
\hline VCR & $1 \mathrm{mg} / \mathrm{m}^{2} \mathrm{IV}$ & Día 1 \\
\hline MTX & $8-15 \mathrm{mg} / \mathrm{m}^{2} \mathrm{IT}$ & Día 1 \\
\hline $\mathrm{HCT}$ & $8-15 \mathrm{mg} / \mathrm{m}^{2} \mathrm{IT}$ & Día 1 \\
\hline CT & $15-30 \mathrm{mg} / \mathrm{m}^{2} \mathrm{IT}$ & Día 1 \\
\hline \multicolumn{3}{|l|}{ Intensificación } \\
\hline MTX AD & $\begin{array}{c}5 \mathrm{~g} / \mathrm{m}^{2} \mathrm{IV} \text { infusión } \\
\text { de } 24 \mathrm{~h}\end{array}$ & Día 1 \\
\hline Doxo & $60 \mathrm{mg} / \mathrm{m}^{2} \mathrm{IV}$ & Día 2 \\
\hline \multicolumn{3}{|l|}{ Consolidación } \\
\hline $\mathrm{CT}$ & $\begin{array}{c}50 \mathrm{mg} / \mathrm{m}^{2} \mathrm{IV} \text { infusión } \\
\text { de } 12 \mathrm{~h}\end{array}$ & Días 1-5 \\
\hline CT-AD & $3 \mathrm{~g} / \mathrm{m}^{2} \mathrm{IV}(3 \mathrm{~h})$ & Días 2-5 \\
\hline VP-16 & $200 \mathrm{mg} / \mathrm{m}^{2} \mathrm{IV}$ & Días 2-5 \\
\hline \multicolumn{3}{|l|}{ Radioterapia } \\
\hline Rt a cráneo & 25 Gy & \\
\hline Rt a neuroeje & 15 Gy & \\
\hline \multicolumn{3}{|c|}{ Segunda línea (similar CYVE) } \\
\hline MTX AD & $\begin{array}{c}5 \mathrm{~g} / \mathrm{m}^{2} \text { Infusión } \\
\text { de } 24 \mathrm{~h}\end{array}$ & Día 1 \\
\hline CT-AD & $\begin{array}{c}2 \mathrm{~g} / \mathrm{m}^{2} \mathrm{IV} 3 \mathrm{~h} \\
\text { (4 dosis) }\end{array}$ & Días 2-5 \\
\hline MTX & $8-15 \mathrm{mg} / \mathrm{m}^{2} \mathrm{IT}$ & Día 1 \\
\hline $\mathrm{HCT}$ & $8-15 \mathrm{mg} / \mathrm{m}^{2} \mathrm{IT}$ & Día 1 \\
\hline CT & $15-30 \mathrm{mg} / \mathrm{m}^{2} \mathrm{IT}$ & Día 1 \\
\hline
\end{tabular}

CFM: ciclofosfamida; PDN: prednisona; VCR: vincristina; HCT: hidrocortisona; MTX: metotrexato; MTX-AD: metotrexato en dosis altas; Doxo: doxorrubicina; CT: citarabina; CT-AD: citarabina en dosis altas; VP-16: etopósido; Rt: radioterapia.

tratecal en pacientes con citología negativa en líquido cefalorraquídeo. ${ }^{9,10,15,21}$

Otro agente que ha demostrado utilidad en el tratamiento de estos linfomas es el arabinósido de citosina (citarabina) a dosis altas $\left(\geq 3 \mathrm{~g} / \mathrm{m}^{2}\right)$ para alcanzar concentraciones activas en sistema nervioso central. ${ }^{2,4,5,8-12,15-18,21}$ En estudios con adultos se ha visto que la asociación de dosis altas, tanto de citarabina como de metotrexato, logró una mayor supervivencia global en comparación con el metotrexato solo; supervivencia global a los dos años de $64 \%$ contra 18\%. ${ }^{5}$ La justificación de
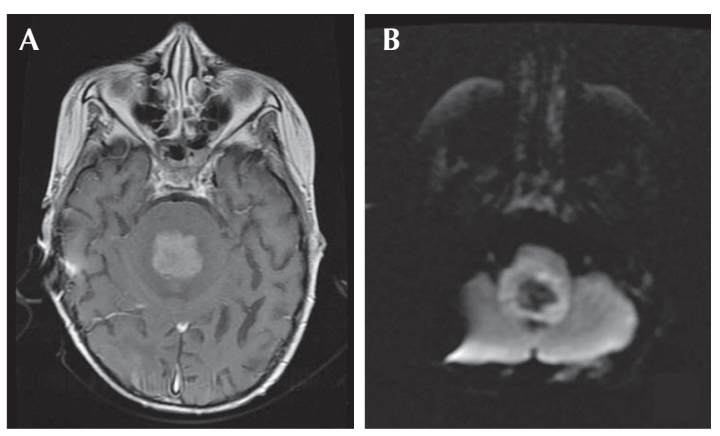

Figura 5. Progresión de la enfermedad. A) Resonancia magnética contrastada en fase T1, corte axial: lesión con realce heterogéneo posterior a la administración del medio de contraste, condiciona obliteración total de la luz del cuarto ventrículo, así como compresión de puente y bulbo raquídeo. B) Resonancia magnética: realce heterogéneo periférico en el patrón de restricción de la difusión.

utilizar dosis altas de estos fármacos se debió a la acción sinérgica que se logra, permaneciendo mayor tiempo de exposición en el sistema nervioso central, lo cual teóricamente beneficia el arresto de las células proliferantes (fase S). ${ }^{5}$ Por otra parte, en algunas series en adultos se han utilizado protocolos de tratamiento basados en BFM-90-NHL en donde se utilizan dosis altas de metotrexato $\left(5 \mathrm{~g} / \mathrm{m}^{2}\right)$ y de citarabina $\left(3 \mathrm{~g} / \mathrm{m}^{2}\right)$ que han mostrado un incremento en la supervivencia a cinco años de $73 \%$; es una de las series con resultados más esperanzadores en relación a la supervivencia. Existen otros estudios como FABLMB-89 y 96 en los que se utilizan dosis altas de metotrexato (hasta $\left.8 \mathrm{~g} / \mathrm{m}^{2}\right)^{9,10,21}$ con beneficio en un pequeño número de pacientes pero cuya toxicidad es muy alta.

Otros medicamentos útiles en el tratamiento de los linfomas son los esteroides (principalmente la dexametasona) que por sí solos han mostrado que pueden inducir una remisión completa aunque transitoria, por lo que actualmente deben considerarse como parte integral del tratamiento. ${ }^{4,5,9,10,12,18,21}$ 
Cárdenas-Cardos $\mathbf{R}$ et al. Linfoma no Hodgkin primario de sistema nervioso central

En nuestra investigación sólo encontramos tres series pequeñas de pacientes en edad pediátrica $^{4,5,15}$ que mencionan buena respuesta al tratamiento y alcanzaron supervivencia de más de $70 \%$ (Cuadro 1); pero la población incluida, la metodología y los tratamientos utilizados fueron diversos y su comparación difícil.

Otra modalidad de tratamiento utilizado en población adulta es la terapia con dosis altas de citotóxicos seguida de un rescate con trasplante de progenitores hematopoyéticos; se mencionan algunos beneficios potenciales del trasplante autógeno de células madre en adultos con enfermedad resistente o recaída; ${ }^{8,11,18}$ la utilidad principal de esta modalidad se da después de esquemas de rescate en los que se utilizan fármacos como temozolomida e incluso topotecan. ${ }^{22,23}$

\section{CONCLUSIÓN}

En las distintas series se ha reportado que la evolución clínica y el pronóstico de los pacientes pediátricos con linfomas primarios del sistema nervioso central son favorables, por lo que es importante destacar el presente caso cuyo curso clínico fue tórpido y con evidencia de progresión a pesar de un tratamiento intenso con esteroide, dosis altas de metotrexato y de citarabina e incluso, a pesar de las recomendaciones de distintas series, radioterapia craneoespinal. Es de suma importancia la identificación temprana y el diagnóstico oportuno de esta afección, así como definir un tratamiento estandarizado para emitir recomendaciones para nuestra población.

\section{CONSIDERACIONES A FUTURO}

El linfoma no Hodgkin primario del sistema nervioso central tiene posibles explicaciones como alteraciones citogenéticas, ${ }^{24}$ la deleción del cromosoma $6 q 22$ y rearreglos del gen bcl6 como indicadores de agresividad. Se han propuesto modelos predictivos ${ }^{6,25}$ pero sólo han sido descritos en población adulta. Sería interesante considerar las concentraciones séricas de lactato-deshidrogenasa, la concentración de proteínas en el líquido cefalorraquídeo y el involucramiento de estructuras profundas del cerebro. Sería necesario estandarizar y buscar dichas alteraciones en nuestra población pediátrica latinoamericana.

\section{REFERENCIAS}

1. Lois $\mathrm{D}$, Ohgaki H, Wiestler O. WHO classification of tumors of the central nervous system. Lyon, France: IARC 2007:189-192.

2. Batchelor T, Loeffler JS. Primary CNS Lymphoma. J Clin Oncol 2006;24:1281-1288.

3. Padhi S, Roshni TP, Challa S. Primary extranodal non Hodgkin lymphoma: a 5 year retrospective analysis. Asian Pacific J Cancer Prev 2012;13:4889-4895.

4. Abla O, Weitzman S, Blay JY, O'Neill BP, Abrey LE, Neuwelt E, et al. Primary CNS Lymphoma in Children and Adolescents: A Descriptive Analysis from the International Primary CNS Lymphoma Collaborative Group (IPCG). Clin Cancer Res 2011;17(2):346-352.

5. O Abla, Sandlund JT, Sung L, Brock P, Corbett R, Kirov I, et al. A Case Series of Pediatric Primary Central Nervous System Lymphoma: Favorable Outcome Without Cranial Irradiation. Pediatr Blood Cancer 2006;47:880-885.

6. Abrey LE, Ben-Porat L, Panageas KS, Yahalom J, Berkey B, Curran W, et al. Primary Central Nervous System Lymphoma: The Memorial Sloan-Kettering Cancer Center Prognostic Model. J Clin Oncol 2006;24:5711-5715.

7. Esptein LG, Frederick J, Dicarlo J, et al. Primary lymphoma of the central nervous system in children with acquired immunodeficiency syndrome. Pediatrics 1988;82:355-363.

8. Jiménez VH, Domínguez V, López-Karpovitch X. Linfoma primario del sistema nervioso central: epidemiología, biología, diagnóstico y tratamiento. Med Int Mex 2007;23:6677.

9. Atra A, Imeson JD, Hobson R, Gerrard M, Hann IM, Eden OB, et al. Improved Outcome in children with advanced stage B-Cell non-Hodgkin's lymphoma (B-NHL): results of the United Kingdom Children Cancer Study Group (UKCCSG) 9002 protocol. Br J Cancer 2000;82:1396-1402.

10. Patte C, Auperin A, Michon J, et al. The Societe Francaise d'Oncologie Pediatrique LMB89 protocol: Highly effective multiagent chemotherapy tailored to the tumor burden and initial response in 561 unselected children with B-cell lymphomas and L3 leukemia. Blood 2001;97:3370-3379.

11. Ferreri A, Abrey L, Blay J, Borisch B, Hochman J, Neuwelt E, et al. Summary Statement on Primary Central Nervous System Lymphomas From the Eighth International Confe- 
rence on Malignant Lymphoma, Lugano, Switzerland, June 12 to 15, 2002 J Clin Oncol 2003;2:2407-2414.

12. Salzburg J, Burkhardt B, Zimmermann M, Wachowski O, Woessmann W, Oschlies I, et al. Prevalence, Clinical Pattern, and Outcome of CNS Involvement in Childhood and Adolescent Non-Hodgkin's Lymphoma Differ by NonHodgkin's Lymphoma Subtype: A Berlin-Frankfurt-Münster Group Report. J Clin Oncol 2007;25:3915-3922.

13. Murphy SB. Classification, staging and end results of treatment of childhood non-Hodgkin's lymphomas: Dissimilarities from lymphomas in adults. Semin Oncol 1980;7:332-339.

14. Reiter A, Schrappe M, Parwaresch R, Henze G, MüllerWeihrich $S$, Sauter $S$, et al. Non-Hodgkin's lymphomas of childhood and adolescence: results of a treatment stratified for biologic subtypes and stage--a report of the BerlinFrankfurt-Münster Group. J Clin Oncol 1995;13:359-72.

15. Hyung JY, Jin HK, Kim H, et al. Successful treatment of primary central nervous system lymphoma without irradiation in children: single center experience. J Korean Med Sci 2012;27:1378-84.

16. Pels H, Schmidt-Wolf IGH, Glasmacher A, Schulz H, Engert A, Diehl V, et al. Primary Central Nervous System Lymphoma: Results of a Pilot and Phase II Study of Systemic and Intraventricular Chemotherapy With Deferred Radiotherapy. J Clin Oncol 2003;21:4489-4495.

17. Angelov L, Doolittle ND, Kraemer DF, Siegal T, Barnett GH, Peereboom DM, et al. Blood-Brain Barrier Disruption and Intra-Arterial Methotrexate-Based Therapy for Newly Diagnosed Primary CNS Lymphoma: A Multi-Institutional Experience. J Clin Oncol 2009;27:3503-3509.

18. Campen CJ, Tombleson RL, Green MR. High-dose chemotherapy with hematopoietic stem cell transplantation for the treatment of primary central nervous system lymphoma. J Neurooncol 2011;101:345-355.

19. Makino K, Nakamura H, Yano S, Kuratsu JI. Pediatric primary CNS lymphoma: longterm survival after treatment with radiation monotherapy. Acta Neurochir (Wien) 2007;149:295-298.

20. Escamilla AG, Cárdenas $C R$, et al. Linfoma no Hodgkin B. en: Rivera-Luna $\mathrm{R}$ editor. Protocolos Técnicos Cáncer en Niños, Consejo Nacional para la Prevención y el Tratamiento del Cáncer en la Infancia y la Adolescencia, 1a ed. México, Editores de Textos Mexicanos S.A. de C.V. 2010, pp. 102-117.

21. Cairo MS, Gerrard M, Sposto R, Auperin A, Pinkerton C, Michon J, et al. Results of a randomized international study of high-risk central nervous system B non-Hodgkin lymphoma and $B$ acute lymphoblastic leukemia in children and adolescents. Blood 2007;109:2736-2743.

22. Omuro A, Taillandier L, Chinot O, Carnin C, Barrie M, Hoang-Xuan K. Temozolomide and methotrexate for primary central nervous system lymphoma in the elderly. J Neurooncol 2007;85:207-211.

23. Voloschin A, Betensky R, Y. Wen P, Hochberg F, Batchelor $T$. Topotecan as salvage therapy for relapsed or refractory primary central nervous system lymphoma. J Neurooncol 2008;86:211-215.

24. Cady FM, O'Neill BP, Law ME, Decker PA, Kurtz DM, Giannini C, et al. Del(6)(q22) and BCL6 Rearrangements in Primary CNS Lymphoma Are Indicators of an Aggressive Clinical Course. J Clin Oncol 2008;26:4814-4819.

25. Ferreri AJM, Blay JY, Reni M, Pasini F, Spina M, Ambrosetti A, et al. Prognostic Scoring System for Primary CNS Lymphomas: The International Extranodal Lymphoma Study Group Experience. J Clin Oncol 2003;21:266-272.

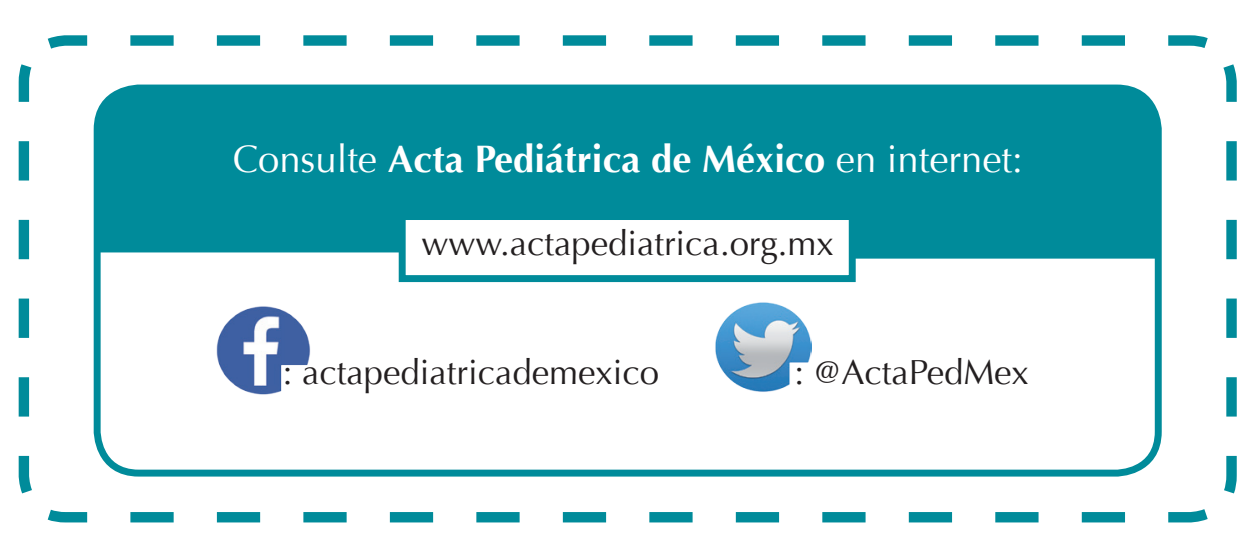

